\title{
MAPT (Tau) expression is a biomarker for an increased rate of survival for low-grade glioma
}

\author{
SAIF ZAMAN $^{1}$, BORIS I. CHOBRUTSKIY ${ }^{1}$, DHIRAJ SIKARIA ${ }^{1}$ and GEORGE BLANCK ${ }^{1,2}$ \\ ${ }^{1}$ Department of Molecular Medicine, Morsani College of Medicine, University of South Florida; \\ ${ }^{2}$ Immunology Program, H. Lee Moffitt Cancer Center and Research Institute, Tampa, FL 33612, USA
}

Received May 15, 2018; Accepted November 8, 2018

DOI: $10.3892 /$ or.2018.6896

\begin{abstract}
The association of MAPT (Tau) with various tauopathies and other neurological disorders has long been established. However, the role of microtubule-associated protein Tau (MAPT) expression in brain cancer is largely unknown. To determine whether MAPT expression is related to low-grade glioma (LGG) survival rates, RNASeq data representing samples from the Cancer Genome Atlas (TCGA) were assessed. Results revealed that high expression of the MAPT gene is very strongly associated with increased overall and disease-free survival in LGG but not in breast cancer or melanoma. No such association was apparent for either amyloid precursor protein or $\alpha$-synuclein gene expression. The expression levels of particular apoptosis- and pro-proliferative-effector genes were consistent with the Tau-associated increased survival rates. It has been well established that the Tau protein plays a neurodegenerative role, and in this study we identified, for the first time, a potential cell apoptosis function that Tau may play in cancers of the central nervous system.
\end{abstract}

\section{Introduction}

The MAPT gene (microtubule-associated protein Tau gene) encodes the Tau protein, which is involved in microtubule

Correspondence to: Dr George Blanck, Department of Molecular Medicine, Morsani College of Medicine, University of South Florida, 12901 Bruce B. Downs Blvd., MDC7, Tampa, FL 33612, USA

E-mail: gblanck@health.usf.edu

Abbreviations: APP, amyloid precursor protein gene; BRCA, breast cancer; CASP9, caspase 9 gene; H2AFX, H2A histone family, member X gene; HIST1H2AL, histone H2A type 1 gene; HIST1H2BK, histone H2B type 1-K gene; HIST1H3J, histone H3J gene; HIST1H4B, histone H4B gene; HIST2H2BE, histone H2B type 2-E gene; HUGO, Human Genome Organization; KM, Kaplan-Meier survival curve; LGG, low-grade glioma; MAPT, microtubule-associated protein Tau; SKCM, skin cutaneous melanoma; SNCA, $\alpha$-synuclein gene; SPSS, IBM Statistical Package for the Social Sciences; TCGA, The Cancer Genome Atlas

Key words: low-grade glioblastoma, MAPT, Tau, apoptosis-effector gene, proliferation-effector gene, survival rate stabilization and tubulin polymerization into microtubules. It has long been established that aggregations of hyperphosphorylated neurofibrillary and gliofibrillary tangles of Tau protein are involved in several neurodegenerative disorders, known as tauopathies $(1,2)$. However, the role of Tau in brain cancers, and specifically, low-grade glioma, has not been explored.

Gliomas are histologically graded from I to IV. Grade I gliomas are usually benign. Grade II [low-grade glioma (LGG)] has an average survival period of $\sim 7$ years. Grade II gliomas can progress into grade III (high-grade gliomas), and eventually grade IV (secondary glioblastoma). Recent research indicates that historically used clinical variables in LGG are inferior prognostic indicators relative to current genetic information, for example the IDH1 mutational status (3). As LGG is an early stage in the progression of gliomas from low grade to high grade, there is a need to understand the disease process from this early onset. Pinpointing key molecular features that are associated with improved outcomes will help elucidate the disease progression and possibly provide prognostic markers (4).

Given Tau's role in neurodegeneration in the brain and microtubule stabilization, it was of interest to explore Tau's connection to LGG. Colodner et al demonstrated that glial fibrillary tangle formation was accompanied by JAK/STAT-mediated apoptotic cell death of both glia and neurons in a Drosophila model of glial tauopathy (5). However, previous research on Tau in the cancer setting, for the most part, has focused on microtubule-inhibiting chemotherapy in breast cancer (6-8). For example, Zhou et al identified Tau expression as a factor to predict tumor sensitivity to microtubule-inhibiting compounds such as taxanes (9). In the present study, we present a novel look into the association of Tau with survival rates for LGG patients.

\section{Materials and methods}

MAPT, apoptosis-effector gene and pro-proliferative gene RNAseq analysis. RNASeq values for MAPT, for the panel of apoptosis-effector genes, and for the panel of pro-proliferative genes, were downloaded from cbioportal.org. Pearson correlation coefficients were obtained, using Microsoft Excel (10-12).

Kaplan-Meier analyses and RNASeq data. The barcodes (patient identifiers) representing 530 LGG samples, 
1,105 BRCA (breast cancer) samples and 478 SKCM (skin cutaneous melanoma) samples, for which RNASeq data were available, were sorted from low to high based on the RNASeq values for each of the indicated genes in the Results section. Survival relationships were then obtained by matching barcode survival data, available from cbioportal.org, with the RNASeq data representing the top and bottom $20 \%$ of the RNASeq values (106 barcodes for LGG, 221 barcodes for BRCA and 94 barcodes for SKCM); and with the top and bottom 50, 40, 30 and $10 \%$ of the RNASeq values for MAPT (LGG only). The clinical data (months of overall survival and disease-free survival) for the associated barcodes were then processed using IBM Statistics Package for the Social Sciences (SPSS), version 24, to provide the Kaplan-Meier (KM) curves and an average difference in survival rates between samples representing the top and bottom RNASeq value percentages (for the genes as indicated in Results).

Histological grade analysis. Histological grades for LGG barcodes were downloaded from cbioportal.org. Histological grade barcodes were then matched to the RNAseq data (106 barcodes for LGG). Comparison of proportions test for the data representing the top and bottom $20 \%$ of the MAPT RNASeq values was carried out using the MedCalc comparison of proportions calculator (https://www.medcalc. org/calc/comparison_of_proportions.php).

Copy number variation (CNV) analysis. Copy numbers for the MAPT gene for LGG barcodes were downloaded from cbioportal.org. Copy numbers were then matched to barcode RNAseq data (530 barcodes for LGG). A Student's t-test for copy number data representing the top and bottom $20 \%$ of the MAPT RNASeq values was carried out using the Microsoft Excel function.

Methylation analysis. Methylation data for the MAPT gene for LGG barcodes were downloaded from cbioportal.org. Methylation data barcodes were then matched to RNAseq data (530 barcodes for LGG). A Student's t-test for the methylation data representing the top and bottom $20 \%$ of the MAPT RNASeq values was carried out using the Microsoft Excel function.

Diagnosis age analysis. Diagnosis age data for LGG barcodes were downloaded from cbioportal.org. Diagnosis age barcodes were then matched to RNAseq data (530 barcodes for LGG). A Student's t-test for diagnosis age representing the top and bottom $20 \%$ of the MAPT RNASeq values was carried out using the Microsoft Excel function.

R2: Genomics Analysis and Visualization Platform. MAPT gene expression and overall survival data for additional glioma datasets were assessed using the 'R2: Genomics Analysis and Visualization Platform (http://r2.amc.nl)'. Single gene Kaplan scan analysis was conducted for MAPT expression (203929_s_at) for each dataset as indicated in Results. Median MAPT mRNA expression was used as a cut-off for overall survival comparisons. The R2 algorithm was used to generate Kaplan-Meier analyses to compare survival outcomes for the top half vs. bottom half of MAPT expressers in each indicated dataset.
Supplementary and supporting material. A large amount of supplementary and supporting documentation has been prepared for the generation of this report, including detailed data supporting for the Materials and methods section directly above. This information is available as a single PDF upon E-mail request to the corresponding author or by accessing: http://www.universityseminarassociates. com/Supporting_online_material_for_scholarly_pubs.php.

\section{Results}

MAPT (Tau) expression and survival rates. We first compared the rate of overall and disease-free survival for LGG patients representing the top and bottom $20 \%$ of MAPT expressers, based on the MAPT RNASeq values representing the TCGA-LGG data set (Fig. 1). This comparison indicated that high MAPT RNASeq values were associated with a significantly increased overall and disease-free survival rates for LGG. To substantiate this result, we compared the top 10, 30, 40 and 50\% MAPT expressers with their corresponding lower level MAPT expressers for overall survival. In all cases, the initial result was confirmed.

To determine whether the above association of MAPT RNASeq levels with the markedly distinct survival rates was specific to $\mathrm{LGG}$, data for overall survival and disease-free survival for BRCA and SKCM tumor barcodes were downloaded from cbioportal.org, and the RNAseq and KM analyses were conducted. Results indicated that MAPT expression levels did not correlate with either overall or disease-free survival rate differences in the cases of BRCA or SKCM (Fig. 1). The mean survival periods and P-values for the log-rank tests are given in the figure legend for Fig. 1. These data indicated that the higher overall and disease-free survival rates for high MAPT expressing patients was specific to LGG.

To determine whether the increased LGG survival rate associated with MAPT expression could be associated with other neurotoxic proteins, we assessed whether significantly increased survival rates could be associated with higher RNASeq values for APP or SNCA. Analyses indicated there were no differences in survival for the patients represented by the LGG barcodes representing the top and bottom quintiles, for RNASeq values for APP or SNCA, respectively (Fig. 2).

LGG apoptosis-effector gene RNASeq values are correlated with MAPT RNASeq values. As Tau is a neurotoxic protein associated with various tauopathies, and contributes to neuronal cell death, we sought to determine whether apoptosis-effector genes were expressed at higher levels in the LGG samples represented by the barcodes with higher levels of MAPT RNASeq values. We first evaluated a set of 28 apoptosis-effector genes (13) for RNAseq expression as represented by the LGG barcodes. Of those 28 genes, we identified a subset of five apoptosis-effector genes that had significantly increased RNASeq values associated with barcodes that represented the top 20\% of MAPT expressors: CASP9, CYC1, CRADD, COX7A2L and UQCRC2 (Table I).

To verify the above positive correlations of apoptosis-effector gene RNASeq values with MAPT RNASeq values, we obtained the Pearson correlation coefficients for the 

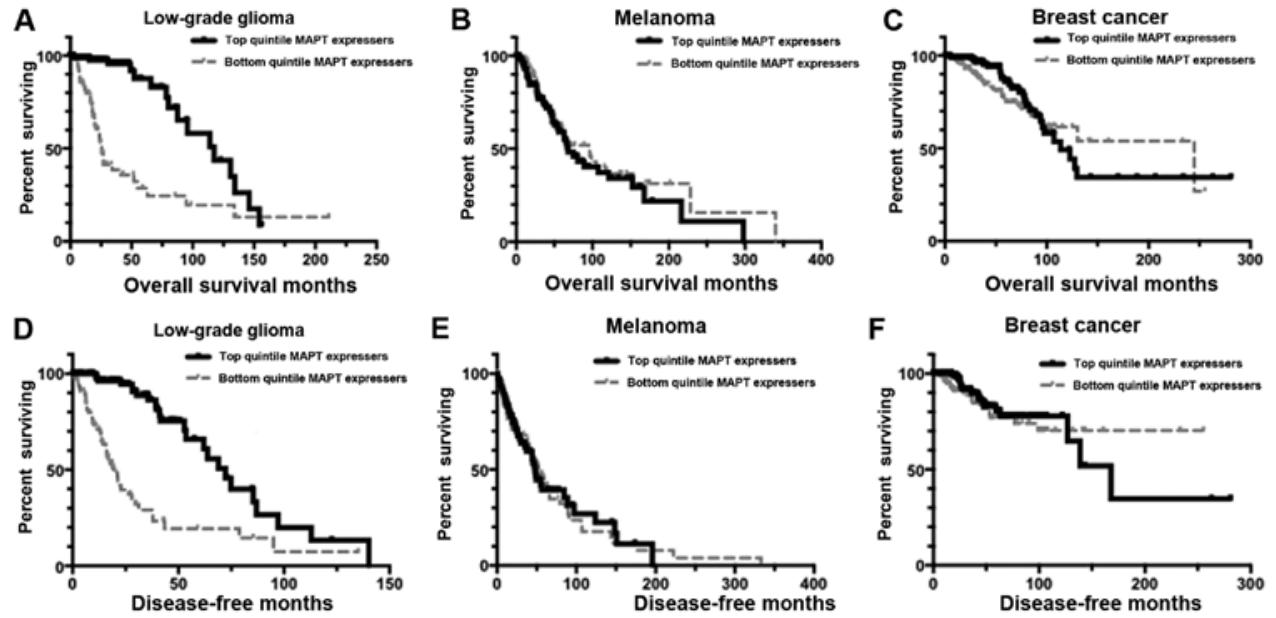

Figure 1. Kaplan-Meier curves representing distinct MAPT expression levels. Results here indicate that there were overall survival (OS) and disease-free survival (DFS) distinctions for low-grade glioma (LGG) based on the MAPT levels in the patient tumors. However, no such distinctions were detectable in BRCA (breast cancer) and SKCM (skin cutaneous melanoma) tumor samples. The following text provides mean survival times and P-values for the Kaplan-Meier (KM) analyses. (A) KM OS curve for LGG barcodes that are in the top quintile of MAPT expressers (n=96), compared to the OS for the bottom quintile of MAPT expressers $(n=104)$. Mean OS for the top quintile of MAPT expressers, 109.04 months; mean OS for bottom quintile of MAPT expressers, 59.36 months. Log-rank comparison P-value, P=9.152E-10. (B) KM OS analysis for SKCM barcodes that are in the top quintile of MAPT expressers (n=90), compared to the OS for the bottom quintile of MAPT expressers $(n=91)$. Mean OS for the top quintile of MAPT expressers, 108.85 months; mean OS for bottom quintile of MAPT expressers, 131.09 months. Log-rank comparison P-value, $\mathrm{P}=0.433$. (C) KM OS analysis for BRCA barcodes that are in the top quintile of MAPT expressers $(n=218)$, compared to the OS for the bottom quintile of MAPT expressers $(n=218)$. Mean OS for the top quintile of MAPT expressers, 154.70 months; mean OS for bottom quintile of MAPT expressers, 164.95 months. Log-rank comparison P-value, P=0.302. (D) KM DFS analysis for LGG barcodes that are in the top quintile of MAPT expressers ( $n=93$ ), compared to the DFS for the bottom quintile of MAPT expressers (n=86). Mean DFS for the top quintile of MAPT expressers, 74.72 months; mean DFS for bottom quintile of MAPT expressers, 34.80 months. Log-rank comparison $\mathrm{P}$-value, $\mathrm{P}=2.215 \mathrm{E}-10$. (E) KM DFS analysis for SKCM barcodes that are in the top quintile of MAPT expressers ( $\mathrm{n}=76$ ), compared to the DFS for the bottom quintile of MAPT expressers ( $\mathrm{n}=80$ ). Mean DFS for the top quintile of MAPT expressers, 71.36 months; mean DFS for bottom quintile of MAPT expressers, 73.59 months. Log-rank comparison P-value, $\mathrm{P}=0.975$. (F) KM DFS analysis for BRCA barcodes that are in the top quintile of MAPT expressers (n=200), compared to the DFS for the bottom quintile of MAPT expressers $(n=198)$. Mean DFS for the top quintile of MAPT expressers, 169.72 months; mean DFS for bottom quintile of MAPT expressers, 192.60 months. Log-rank comparison P-value, $\mathrm{P}=0.316$. MAPT, microtubule-associated protein Tau gene.
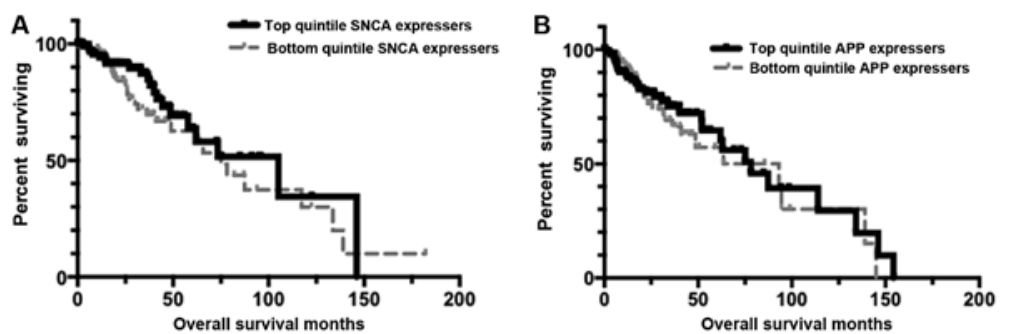

Figure 2. Kaplan-Meier (KM) curves representing distinct APP and SNCA expression levels. Results here indicate that no survival distinctions are detectable for low-grade glioma (LGG) based on APP or SNCA expression levels. (A) KM overall survival (OS) analysis for LGG barcodes that are in the top quintile of SNCA expressers $(n=105)$, compared to the OS for the bottom quintile of SNCA expressers $(n=100)$. Mean OS for the top quintile of MAPT expressers, 89.44 months; mean OS for bottom quintile of MAPT expressers, 82.74 months. Log-rank comparison P-value, $\mathrm{P}=0.279$. (B) KM OS analysis for LGG barcodes that are in the top quintile of APP expressers $(n=102)$, compared to the OS for the bottom quintile of APP expressers ( $n=102)$. Mean OS for the top quintile of MAPT expressers, 134.17 months; mean OS for bottom quintile of MAPT expressers, 138.93 months. Log-rank comparison $\mathrm{P}$-value, $\mathrm{P}=0.565$. APP, amyloid precursor protein gene; MAPT, microtubule-associated protein Tau gene.

Table I. Average apoptosis-effector gene RNASeq values for LGG barcodes representing high and low expression of MAPT.

\begin{tabular}{lccr}
\hline Apoptosis-effector gene & Bottom 20\% MAPT RNASeq values & Top 20\% MAPT RNASeq values & P-value \\
\hline CASP9 & 511.0 & 901.2 & $<0.0001$ \\
COX7A2L & $1,158.2$ & $1,300.2$ & 0.0011 \\
CRADD & 193.1 & 215.0 & 0.0054 \\
CYC1 & $1,952.8$ & $2,339.4$ & $<0.0001$ \\
UQCRC2 & $2,601.8$ & $3,853.1$ & $<0.0001$ \\
\hline
\end{tabular}

LGG, low-grade glioma; MAPT, microtubule-associated protein Tau gene; CASP9, caspase 9; COX7A2L, cytochrome $c$ oxidase subunit 7A2 like; CRADD, CASP2 and RIPK1 domain containing adaptor with death domain; CYC1, cytochrome $c 1$; UQCRC2, ubiquinol-cytochrome $c$ reductase core protein 2 . 

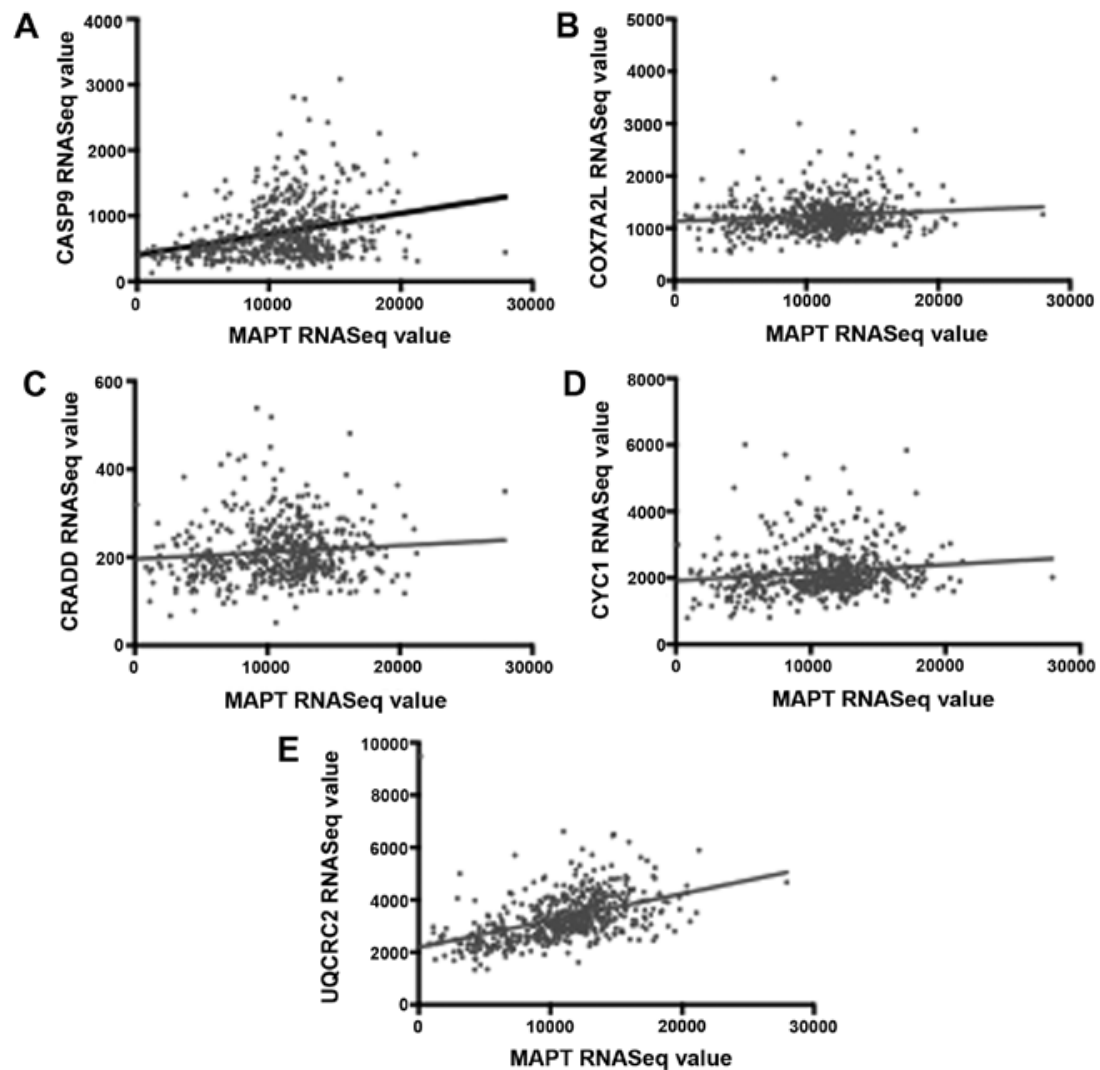

Figure 3. Correlation of MAPT and apoptosis-effector gene expression levels. Results here indicate correlation of high MAPT expression levels with high apoptosis-effector gene expression levels, possibly providing an explanation for the longer survival rates observed with higher MAPT levels. (A) MAPT vs. CASP9 (B) MAPT vs. COX7A2L (C) MAPT vs. CRADD (D) MAPT vs. CYC1 (E) MAPT vs. UQCRC2. The P-values for the Pearson correlation coefficients are $\mathrm{P}<0.00001, \mathrm{P}=0.00723, \mathrm{P}=0.02863, \mathrm{P}=0.00169$ and $\mathrm{P}<0.00001$, respectively for each relationship. MAPT, microtubule-associated protein Tau gene; CASP9, caspase 9; COX7A2L, cytochrome $c$ oxidase subunit 7A2 like; CRADD, CASP2 and RIPK1 domain containing adaptor with death domain; CYC1, cytochrome $c 1$; UQCRC2, ubiquinol-cytochrome $c$ reductase core protein 2 .
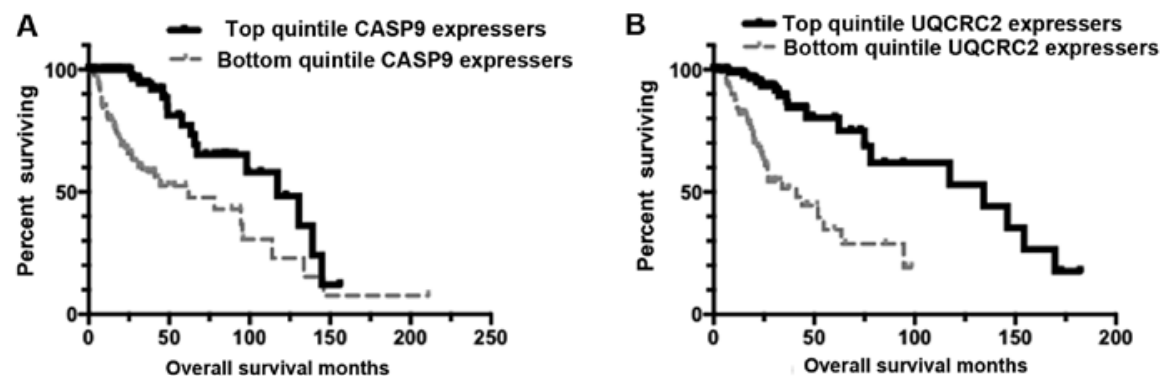

Figure 4. Kaplan-Meier (KM) curves representing distinct apoptosis-effector gene expression levels. Results presented here indicate that apoptosis-effector gene expression represents an independent marker of survival rates. (A) KM overall survival (OS) curve for low-grade glioma (LGG) barcodes that are in the top quintile of CASP9 expressers $(n=100)$, compared to the OS for the bottom quintile of CASP9 expressers $(n=105)$. Mean OS for the top quintile of CASP9 expressers, 105.309 months; mean OS for bottom quintile of CASP9 expressers, 73.471 months. Log-rank comparison P-value, P=0.000014. (B) KM OS curve for LGG barcodes that are in the top quintile of UQCRC2 expressers ( $\mathrm{n}=103$ ), compared to the OS for the bottom quintile of UQCRC2 expressers ( $\mathrm{n}=103$ ). Mean OS for the top quintile of UQCRC2 expressers, 115.495 months; mean OS for bottom quintile of UQCRC2 expressers, 48.623 months. Log-rank comparison P-value, $\mathrm{P}=1.971 \mathrm{E}-7$. CASP9, caspase 9; MAPT, microtubule-associated protein Tau gene; UQCRC2, ubiquinol-cytochrome $c$ reductase core protein 2.

MAPT RNA expression levels and the RNA expression levels of CASP9, CYC1, CRADD, COX7A2L and UQCRC2 in LGG. In each case, there was a statistically significant correlation with MAPT expression (Fig. 3).

To determine whether any of the five above identified apoptosis-effector genes represented independent markers of survival rates, the overall survival data for LGG barcodes representing the highest $20 \%$ and lowest $20 \%$ of CASP9, CYC1, CRADD, COX7A2L and UQCRC2 expression levels were obtained for KM analysis. Results indicated that only CASP9 and UQCRC2 represented independent markers of survival, with barcodes representing the highest $20 \%$ of both CASP9 and UQCRC2 having significantly increased survival rates (Fig. 4).

$L G G$ proliferation-effector gene RNASeq values are correlated with MAPT RNASeq values. We next sought to determine whether any proliferation-effector genes were 


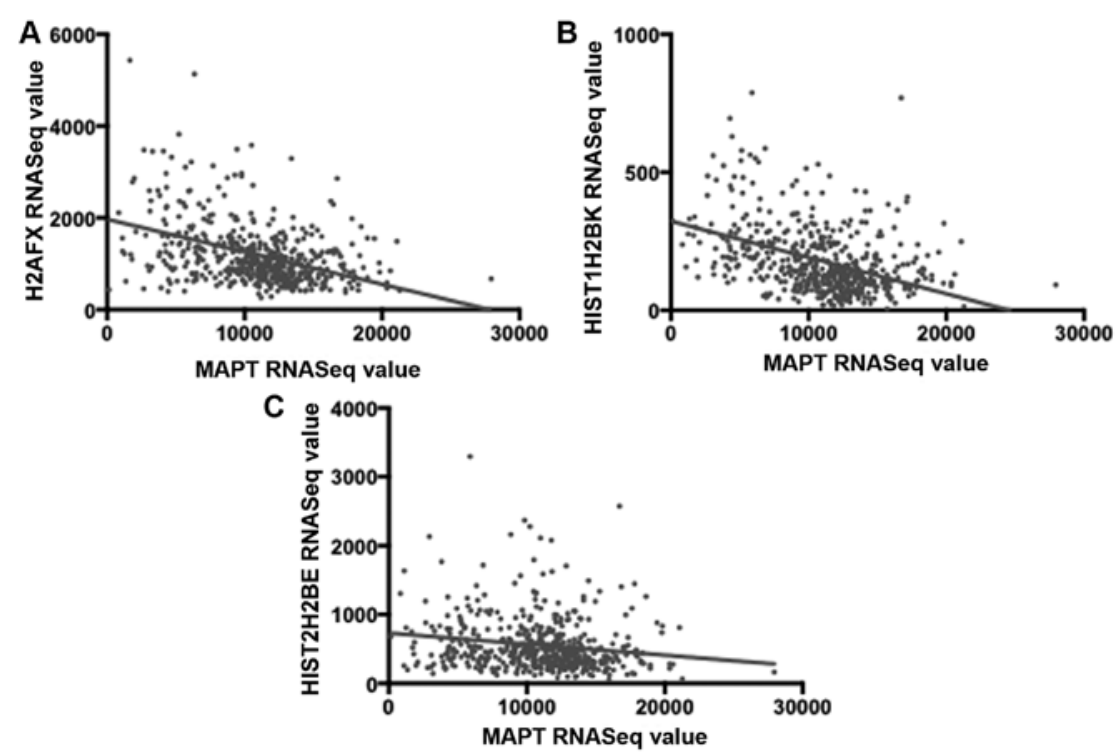

Figure 5. Inverse correlation of MAPT and proliferation-effector gene expression levels. Results presented here were consistent with increased tumor cell replication in cases of lower MAPT expression and reduced survival rates. (A) MAPT vs. H2AFX (B) MAPT vs. HIST1H2BK (C) MAPT vs. HIST2H2BE. The $\mathrm{P}$-values for the Pearson correlation coefficient are $\mathrm{P}<0.00001, \mathrm{P}<0.00001$, and $\mathrm{P}=0.000727$, respectively for each relationship. MAPT, microtubule-associated protein Tau gene; H2AFX, H2A histone family, member X gene; HIST1H2BK, histone H2B type 1-K gene; HIST2H2BE, histone H2B type 2-E gene.

Table II. Identifying proliferation-effector gene survival associations in LGG..

\section{Gene}

Survival distinctions

$\mathrm{H} 2 \mathrm{AFX}$ HIST1H2BK

HIST2H2BE

Increased/decreased survival for the top quintile

Decreased

Decreased

Decreased

of expressers of the indicated gene

Increased/decreased survival for the bottom

Increased

Increased

Increased

quintile of expressers of the indicated gene

KM, log-rank P-value

$<0.0001$

$<0.0001$

$<0.0001$

LGG, low-grade glioma; H2AFX, H2A histone family, member X gene; HIST1H2BK, histone H2B type 1-K gene; HIST2H2BE, histone H2B type 2-E gene; KM, Kaplan-Meier.

expressed at higher levels in the samples with lower levels of the MAPT RNASeq values. We previously established a panel of 62 proliferation-effector genes (13). Of those 62 genes, we identified a subset of 28 proliferation-effector genes that have significantly increased RNASeq expression in barcodes that represent the bottom $20 \%$ of MAPT expressers.

To verify the above inverse correlations of proliferation-effector gene expression with MAPT expression, we obtained the Pearson correlation coefficients for MAPT RNASeq values and the RNASeq values for a histone subset of the above indicated, 28 proliferation-effector genes, for the LGG barcodes: H2AFX, HIST1H2BK and HIST2H2BE. In each case, there was a statistically significant inverse correlation with MAPT expression (Fig. 5).

To determine whether any of the above indicated histone genes represented independent survival markers, we generated KM survival curves based on the top and bottom LGG expressers. From these analyses, we determined that the expression of all three of the histone genes independently represented survival distinctions (Table II).

LGG histological grade is correlated with MAPT RNASeq values. To determine whether MAPT expression correlates with histological features of $\mathrm{LGG}$, we compared histological grades of the top quintile and bottom quintile of MAPT-expressing barcodes. All LGG barcodes in the dataset used were graded either G2 or G3. We determined that the low MAPT-expressing barcodes, that also represented decreased survival rates, also had a greater proportion of G3 histological grade than the high MAPT-expressing barcodes (Table III).

$C N V$ and methylation are correlated with MAPT RNASeq values in $L G G$. To determine whether there may be any genetic or epigenetic associations with increased MAPT expression, we compared MAPT copy number variation (CNV) and methylation of the MAPT gene in the top quintile and bottom quintile of MAPT-expressing barcodes using data available on 
Table III. Identification of histological grade associations with MAPT expression.

\begin{tabular}{lccc}
\hline & Bottom quintile MAPT expressers & Top quintile MAPT expressers & P-value \\
\hline Fraction G3 histological grade & 0.78 & 0.22 & $<0.0001$ \\
\hline
\end{tabular}

MAPT, microtubule-associated protein Tau gene.

Table IV. Copy number variation and methylation associations with MAPT expression.

\begin{tabular}{lccc}
\hline & Bottom quintile MAPT expressers & Top quintile MAPT expressers & P-value \\
\hline Copy no. variation & -0.031 & 0.048 & 0.0002 \\
Methylation & 0.490 & 0.355 & $3.479 \mathrm{E}-13$ \\
\hline
\end{tabular}

MAPT, microtubule-associated protein Tau gene.

Table V. Diagnosis age associations with MAPT expression.

\begin{tabular}{lccc}
\hline & Bottom quintile MAPT expressers & Top quintile MAPT expressers & P-value \\
\hline Average age of diagnosis & 48.56 & 39.38 & $4.248 \mathrm{E}-07$ \\
\hline
\end{tabular}

MAPT, microtubule-associated protein Tau gene.

Table VI. Identifying MAPT expression associations with survival in additional glioma datasets, using the R2: Genomics Analysis and Visualization Platform.

Dataset

\begin{tabular}{|c|c|c|}
\hline Survival distinctions & $\begin{array}{l}\text { Glioma (French) } \\
\mathrm{n}=284\end{array}$ & $\begin{array}{l}\text { Glioma (Kawaguchi) } \\
\qquad \mathrm{n}=50\end{array}$ \\
\hline $\begin{array}{l}\text { Increased/decreased survival for the top half } \\
\text { of MAPT expressers in the indicated dataset }\end{array}$ & Increased & Increased \\
\hline $\begin{array}{l}\text { Increased/decreased survival for the bottom half } \\
\text { of MAPT expressers in the indicated dataset }\end{array}$ & Decreased & Decreased \\
\hline KM, log-rank P-value & $<0.0001$ & $<0.001$ \\
\hline
\end{tabular}

MAPT, microtubule-associated protein Tau gene; KM, Kaplan-Meier.

cbioportal.org. For CNV, a negative result indicates average copy number loss, while a positive result indicates average copy number gain among the barcodes analyzed. Our results indicated that there is an average increase in MAPT copy number among barcodes that are in the top quintile of MAPT expressers, while there is an average decrease in copy number among barcodes that are in the bottom quintile of MAPT expressers. Furthermore, our results indicated that there is an increase in MAPT gene methylation among barcodes that are in the bottom quintile of MAPT expressers (Table IV). Together, the CNV and methylation results indicated that there are likely straightforward genetic and epigenetic explanations for decreased MAPT expression.
Diagnosis age is correlated with MAPT RNASeq values in $L G G$. To determine whether MAPT expression may have an effect on diagnosis age, we compared the average age of diagnosis in patient barcodes representing the top and bottom quintile of MAPT RNASeq expression using data available on cbioportal.org. Results indicated that increased MAPT expression was associated with an earlier diagnosis age. The top quintile of MAPT expressers had an average diagnosis age of 39, while the lowest quintile of MAPT expressers had an average diagnosis age of 48 (Table $\mathrm{V}$ ).

Analysis of MAPT expression and survival in additional glioma datasets from the R2: Genomics Analysis and 
Visualization Platform. To determine if MAPT mRNA expression is associated with improved survival outcomes in additional, independent glioma datasets, the R2: Genomics analysis and visualization platform was used. Two additional glioma datasets were analyzed, French $(n=284)$ and Kawaguchi $(n=50)$. In both datasets, the top half of MAPT expressers had improved overall survival outcomes when compared to the bottom half of MAPT expressers (Table VI). Thus, results from these datasets were consistent with the above analyses of the TCGA LGG dataset.

\section{Discussion}

In summary, the above data and analyses indicated that Tau expression was associated with a dramatic increase in survival in low-grade glioma (LGG) patients. While the Tau mechanism in LGG is unknown, we demonstrated that increased Tau expression was correlated with an increase in the expression of several apoptosis-effector genes; and that lack of Tau was associated with a higher level of proliferation-effector gene expression. In addition, the histological grade of the tumor was inversely correlated with Tau expression.

The precise process of Tau-induced neurotoxicity in tauopathies also remains a mystery, but may be due to multiple causes, including DNA damage. In neurons, Tau aggregates become misfolded and hyperphosphorylated, leading to the bundling and stabilization of filamentous actin, ultimately causing oxidative stress in the cell through dysfunctional mitochondria. The oxidative stress causes DNA damage and causes the loss of heterochromatin, allowing genes that are normally silenced by heterochromatin to be transcribed, ultimately leading to cell cycle activation and apoptosis in mature neurons (14). Several studies have noted the link between DNA damage and neurodegeneration in Alzheimer's disease (15-19).

While much has been explored regarding the role of Tau pathology in neurons, there has been less focus on investigating the role of Tau aggregation in glial cells. Several studies have demonstrated the functional consequences of Tau aggregation in glial cells $(5,20)$. Various studies indicate that Tau pathology in glial cells is similar to that in neurons, to an extent, but is not identical $(21,22)$. If we apply the model of Tau oligomerization impact on neural cells (in tauopathies), to glial cells, Tau accumulation in glial cells (in LGG) could trigger a DNA damage response and apoptosis. Our results would be consistent with this hypothesis through increased expression of several apoptosis-effector genes in the population of patients that have high MAPT expression.

Tau is crucial in stabilizing and organizing axonal microtubules in neural cells (23). However, in various tauopathies, Tau forms hyperphosphorylated aggregates which causes the disruption of the microtubule network (24). While the role of Tau in glial cells remains less-investigated, if we apply the previously established model of Tau aggregation and phosphorylation contributing to microtubule destabilization in neural cells to glial cells, it becomes evident that decreased Tau aggregation may allow increased microtubule stabilization, and thus increased cell proliferation. The above data are consistent with this idea as LGG samples with decreased MAPT expression expressed higher proliferation-effector genes, and specifically expressed more histone genes.

\section{Acknowledgements}

Authors acknowledge the financial support of the taxpayers of the State of Florida; and would also like to thank Laura Blair for the helpful discussions.

\section{Funding}

No specific funding was received.

\section{Availability of data and materials}

The supporting online material files indicated in the article are available upon E-mail reqeust to the corresponding author.

\section{Authors' contributions}

SZ made the initial discovery connecting Tau expression to LGG survival outcomes and conducted the majority of the subsequent analyses. BIC conducted the analyses regarding the proliferation-effector genes. DS contributed to the design of particular aspects of RNASeq interpretations. GB supervised the project, provided guidance for the design of the project, conducted some of the analyses directly, spot-checked much of the analyses, wrote most of the drafts of the manuscript and takes full responsibility for the content. All authors read and approved the manuscript and agree to be accountable for all aspects of the research in ensuring that the accuracy or integrity of any part of the work are appropriately investigated and resolved.

\section{Ethics approval and consent to participate}

Not applicable

\section{Patient consent for publication}

Not applicable.

\section{Competing interests}

The authors state that they have no competing interests.

\section{References}

1. Spillantini MG and Goedert M: Tau pathology and neurodegeneration. Lancet Neurol 12: 609-622, 2013.

2. Ikeda K, Akiyama H, Arai T and Nishimura T: Glial tau pathology in neurodegenerative diseases: Their nature and comparison with neuronal tangles. Neurobiol Aging 19 (Suppl): S85-S91, 1998.

3. Hartmann C, Hentschel B, Tatagiba M, Schramm J, Schnell O, Seidel C, Stein R, Reifenberger G, Pietsch T, von Deimling A, et al: Molecular markers in low-grade gliomas: predictive or prognostic? Clin Cancer Res 17: 4588-4599, 2011.

4. Claus EB, Walsh KM, Wiencke JK, Molinaro AM, Wiemels JL, Schildkraut JM, Bondy ML, Berger M, Jenkins R and Wrensch M: Survival and low-grade glioma: The emergence of genetic information. Neurosurg Focus 38: E6, 2015.

5. Colodner KJ and Feany MB: Glial fibrillary tangles and JAK/STAT-mediated glial and neuronal cell death in a Drosophila model of glial tauopathy. J Neurosci 30: 16102-16113, 2010.

6. Li ZH, Xiong QY, Tu JH, Gong Y, Qiu W, Zhang HQ, Wei WS, Hou YF and Cui WQ: Tau proteins expressions in advanced breast cancer and its significance in taxane-containing neoadjuvant chemotherapy. Med Oncol 30: 591, 2013. 
7. Koo DH, Lee HJ, Ahn JH, Yoon DH, Kim SB, Gong G, Son BH, Ahn SH and Jung KH: Tau and PTEN status as predictive markers for response to trastuzumab and paclitaxel in patients with HER2-positive breast cancer. Tumour Biol 36: 5865-5871, 2015.

8. Wang K, Deng QT, Liao N, Zhang GC, Liu YH, Xu FP, Zu J, Li XR and Wu YL: Tau expression correlated with breast cancer sensitivity to taxanes-based neoadjuvant chemotherapy. Tumour Biol 34: 33-38, 2013.

9. Zhou J, Qian S, Li H, He W, Tan X, Zhang Q, Han G, Chen G and Luo R: Predictive value of microtubule-associated protein Tau in patients with recurrent and metastatic breast cancer treated with taxane-containing palliative chemotherapy. Tumour Biol 36: 3941-3947, 2015

10. Grossman RL, Heath AP, Ferretti V, Varmus HE, Lowy DR, Kibbe WA and Staudt LM: Toward a shared vision for cancer cenomic data. N Engl J Med 375: 1109-1112, 2016.

11. Gao J, Aksoy BA, Dogrusoz U, Dresdner G, Gross B, Sumer SO Sun Y, Jacobsen A, Sinha R, Larsson E, et al: Integrative analysis of complex cancer genomics and clinical profiles using the cBioPortal. Sci Signal 6: pl1, 2013.

12. Ping Z, Siegal GP, Almeida JS, Schnitt SJ and Shen D: Mining genome sequencing data to identify the genomic features linked to breast cancer histopathology. J Pathol Inform 5: 3, 2014.

13. Mauro JA and Blanck G: Functionally distinct gene classes as bigger or smaller transcription factor traps: A possible stochastic component to sequential gene expression programs in cancer. Gene 536: 398-406, 2014.

14. Frost B, Götz J and Feany MB: Connecting the dots between tau dysfunction and neurodegeneration. Trends Cell Biol 25: 46-53, 2015.

15. Iijima-Ando K, Zhao L, Gatt A, Shenton C and Iijima K: A DNA damage-activated checkpoint kinase phosphorylates tau and enhances tau-induced neurodegeneration. Hum Mol Genet 19 : $1930-1938,2010$

16. Khurana V, Merlo P, DuBoff B, Fulga TA, Sharp KA Campbell SD, Götz J and Feany MB: A neuroprotective role for the DNA damage checkpoint in tauopathy. Aging Cell 11: $360-362,2012$
17. Kruman II, Wersto RP, Cardozo-Pelaez F, Smilenov L, Chan SL, Chrest FJ, Emokpae R Jr, Gorospe M and Mattson MP: Cell cycle activation linked to neuronal cell death initiated by DNA damage. Neuron 41: 549-561, 2004.

18. Silva AR, Santos AC, Farfel JM, Grinberg LT, Ferretti RE, Campos AH, Cunha IW, Begnami MD, Rocha RM, Carraro DM, et al: Repair of oxidative DNA damage, cell-cycle regulation and neuronal death may influence the clinical manifestation of Alzheimer's disease. PLoS One 9: e99897, 2014.

19. Simpson JE, Ince PG, Matthews FE, Shaw PJ, Heath PR, Brayne C, Garwood C, Higginbottom A, Wharton SB and Function MRCC; MRC Cognitive Function and Ageing Neuropathology Study Group: A neuronal DNA damage response is detected at the earliest stages of Alzheimer's neuropathology and correlates with cognitive impairment in the Medical Research Council's Cognitive Function and Ageing Study ageing brain cohort. Neuropathol Appl Neurobiol 41: 483-496, 2015.

20. Nishimura M, Tomimoto H, Suenaga T, Namba Y, Ikeda K, Akiguchi I and Kimura J: Immunocytochemical characterization of glial fibrillary tangles in Alzheimer's disease brain. Am J Pathol 146: 1052-1058, 1995.

21. Ferrer I, López-González I, Carmona M, Arregui L, Dalfó E, Torrejón-Escribano B, Diehl R and Kovacs GG: Glial and neuronal tau pathology in tauopathies: Characterization of disease-specific phenotypes and tau pathology progression. J Neuropathol Exp Neurol 73: 81-97, 2014.

22. Arai T, Ikeda K, Akiyama H, Shikamoto Y, Tsuchiya K, Yagishita S, Beach T, Rogers J, Schwab C and McGeer PL: Distinct isoforms of tau aggregated in neurons and glial cells in brains of patients with Pick's disease, corticobasal degeneration and progressive supranuclear palsy. Acta Neuropathol 101: 167-173, 2001.

23. Harada A, Oguchi K, Okabe S, Kuno J, Terada S, Ohshima T, Sato-Yoshitake R, Takei Y, Noda T and Hirokawa N: Altered microtubule organization in small-calibre axons of mice lacking tau protein. Nature 369: 488-491, 1994

24. Mandelkow E and Mandelkow EM: Microtubules and microtubule-associated proteins. Curr Opin Cell Biol 7: 72-81, 1995. 